\title{
Evaluation of Emissions from Delayed Departure Flights at Bandaranaike International Airport (BIA)
}

\author{
D.M.M.S. Dissanayaka, V. Adikariwattage, and H.R Pasindu
}

\begin{abstract}
Avoiding flight delays has become a crucial factor in each airport and airline since a huge economic cost results as consequences. Researches are conducted to measure aviation delay and its economic cost [1] while its environmental cost is sparsely addressed. In this research, the delay of departure flights at ground operations are referred when waiting to takeoff. More emissions arouse than usual from excess fuel consumption when a flight experiences delay. A methodology is developed in this research to evaluate the impact of departure flight delays at ground operations. Bandaranaike International Airport (BIA) is taken as a case for developing the methodology.
\end{abstract}

Index Terms-Departure flight delay, emission, ACERT, unimpeded taxi-out time, Taxi-out time delay.

\section{INTRODUCTION}

$\mathrm{B}_{\mathrm{t}}$ eing relatively safer transport mode, the demand for air transport shows a rapid growth. Overtaking the 7 billion air passenger mark in 2015, the global air traffic is expected to double by 2029 with the growth rate of $5.2 \%$ per annum [2].

Aviation contributes $2 \%$ to the total Greenhouse gas (GHG) emission [3]. By 2020, the global aviation emissions are forecasted to be around $70 \%$ higher than in 2005. By 2050, aviation emission is forecasted to grow by further $300-700 \%$ [4]. With the notable boom in air transport operations, the aviation sector is confronting a global issue of climate change. International civil aviation should follow rules and regulations issued by the International Civil Aviation Organization (ICAO) to achieve safety standards. However, ICAO sets goals in the environmental aspect which are not even mandatory that all aviation stakeholders should adhere. Few airports have voluntarily adopted eco-friendly initiatives.

\section{BACKGROUND}

Arrival flights are received priority over departure flights at each airport since the airborne delay is more costly and riskier

D.M.M.S. Dissanayaka is with the Department of Transport and Logistics Management, University of Moratuwa, Sri Lanka (e-mail: mano90diss@gmail.com).

V. Adikariwattage is with the Department of Transport and Logistics Management, University of Moratuwa, Sri Lanka (e-mail: varunacric2000@gmail.com).

H.R Pasindu is with the Department of Civil Engineering, University of Moratuwa, Sri Lanka (e-mail: h.r.pasindu@gmail.com), compared to a ground delay which is more easily managed [5]. Whenever the flight demand is increased runway capacity, the flight delay can be expected. Then, some aircraft are asked to hold in the air till the runway gets its clearance while some aircraft are asked to be in taxiways till the runway gets its clearance.

The delay of an aircraft carries the ripple effect. When an aircraft delays it affects to the other aircraft in a queue of landing or taking off. Therefore, one flight delay can carry a huge impact on the number of stakeholders. When considering departure flight delays; the gates, apron area, taxiways and runways are the areas where congestion could occur in ground movement operation of a flight.

Taxi-out time is defined as the time between the point at which a flight actually pushes back from its gate and the time at which it is cleared for take-off. Taxi-out times depend on number of factors such as the active runway (the wind direction or time of day), the distance from the gate to the runway, weather (de-icing needed or low visibility), congestion levels (length of the queue to take-off), apron/taxiway layout and aircraft type (wake vortex).

Taxiing delay is the additional waiting time caused by traffic flow management constraints which make the flight wait or queue. Unimpeded taxi-out time is measured in order to calculate actual taxi-out delay and its emission. Unimpeded taxi-out time is defined as the time between the point where an aircraft pushes back from the gate and takeoff from the runway without any interference. This time component is considered as the reference to estimate flight delays during the taxi-out phase. U.S. and European systems follow the same definition of unimpeded taxiing time [6].

Flight delays have a significant impact on the environment as it burns additional aircraft fuel than expected and causes for more carbon emission. While minimizing flight delays, fuel efficiency can be improved and carbon emission can be reduced. Taxi-out fuel consumption is mostly calculated with the fuel burn indices presented in the International Civil Aviation Organization (ICAO) engine emissions databank [7].

Airport Council International (ACI) has introduced the Airport Carbon and Emissions Reporting Tool (ACERT) to convert the fuel usage to emissions at the airport operations. Methodologies of calculating emissions in ACERT are consistent with the ACI Guidance Manual on Airport Greenhouse Gas Emissions Management (2009) and the GHG 
Protocol. This tool has been tested at several major airports including Zurich, Toronto and SeaTac [8].

Bandaranaike International Airport (IATA: CMB, ICAO: VCBI) is the main international airport serving Sri Lanka. Being an ideal capacity constrained airport, BIA presently handles over 9.8 million passengers per annum, although the designed handling capacity of the terminal building stands at 6 million [9]. With the rapid growth in air traffic volume, BIA and airspace system are under increasing pressure, which leads to frequent flight delays.

BIA consists with 5 taxiways (A, B, C, D and E) TWY A and $\mathrm{E}$ are situated $120 \mathrm{~m}$ from runway Centre line while taxiways $\mathrm{B}, \mathrm{C}$ and $\mathrm{D}$ are situated $90 \mathrm{~m}$ away from runway Centre line. BIA operates 24 hours and runway 04/22 is closed between 0845-1115 (GMT) on every Wednesday for maintenance.

The key objective of this paper is to estimate annual $\mathrm{CO}_{2}$ emission aroused due to departure flight delays. The above main objective is achieved by analyzing departure data of BIA to calculate the flight delays due to congestion at the movement area and assessing additional fuel burn due to delay.

\section{Methodology}

Departure flight data at BIA were observed for this study. The information was extracted from the Daily Aircraft Movement Record (DAMR) which is maintained by Air Traffic Controllers (ATC). The database contains information such as Aircraft type, movement type (arrival, departure, touch \& go), take-off time/ landing time, the category of flight (scheduled, non- scheduled), gate departure/ gate arrival time and parking bay are the information received from the database. Fig.1.depicts the research framework used for the study.

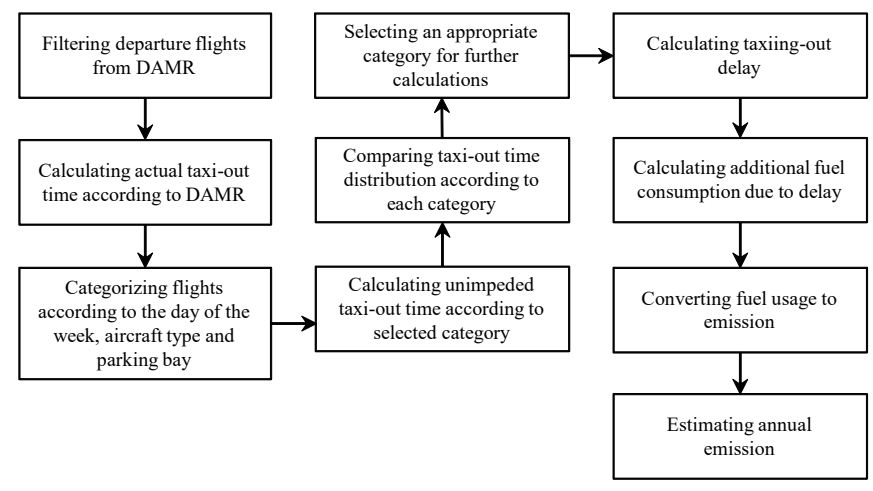

Fig. 1. Research Framework

Fig. 2 depicts the steps followed by a departure flight before the take -off. Following phases of an aircraft are considered in this research in order to identify activities which cause for departure flight delays at ground operations.

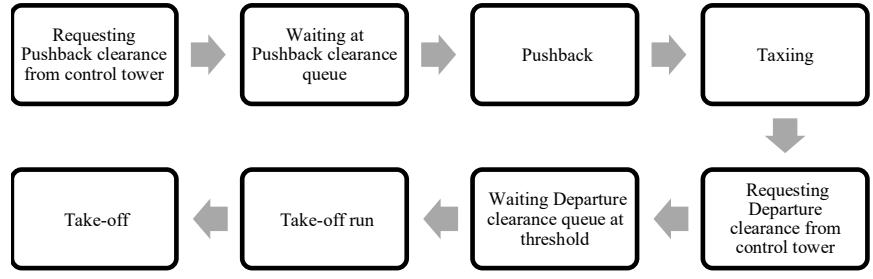

Fig. 2. Steps followed by a departure flight at ground operations

\section{A. Taxi-out time calculation}

Actual taxi-out time was calculated using the difference between take-off time and gate departure.

Actual Taxi out time $=$ Take-off time - Gate departure time (1)

According to the equation 1, the time spent for pushback phase is also included in taxi-out time even it does not belong to that phase. In this research, the time spent on pushback is also considered as the taxi-out time due to unavailability of data. The time spent on pushback is not recorded at BIA.

\section{B. Taxi out Delay calculation}

The taxi-out delay is calculated using the difference between the actual taxi-out time and the unimpeded taxi-out time of the flight.

Taxi-out delay $=$ Actual Taxi-out time - Unimpeded taxi out time

\section{Annual Emission estimation}

Flight schedules at BIA do not significantly vary over the months [10]. Therefore, it is assumed that the flight mix is also consistent throughout the year at BIA. Monthly total aircraft mix was calculated as the actual aircraft movement data is available for each month [10].

Fuel usage for taxiing per minute and emission aroused in taxiing per minute according to the aircraft engine types are given in the ACERT tool. That was used in this research for calculating additional fuel consumption and emission at the taxiing phase.
Excess fuel $=$ Delay in taxi-out $*$ taxiing out fuel usage per minute usage per flight per flight according to engine type

Emission $=$ fuel usage $*$ emission from taxiing per minute per flight according to engine type

\section{Results AND ANALYsis}

\section{A. Calculation of Taxi-out time}

According to equation 1, actual taxi-out time was calculated. The taxi-out time of all the observed departure flights was categorized according to the day of the week, parking aprons(A, B, C and D) and aircraft types (Heavy, Light, Medium). Then the taxi-out time distributions under 
those different categories were compared using ANOVA to detect any significant differences.

Taxi-out time distributions under each category do not depict a significant difference in sample means of the actual taxi-out time.

\section{B. Deciding unimpeded Taxi-out time}

Taxi-out time according to aircraft type was considered in deciding unimpeded taxi-out time for this study. Because fuel consumption at taxiing and emission at taxiing depend on the aircraft engine type.

Therefore, unimpeded taxi-out times were decided according to heavy, medium and light aircraft. The fig. 3, 4 and 5 show distributions of actual taxi out time according to the flight types (Heavy, light and medium). It is assumed that the $15^{\text {th }}$ percentile from the total distribution taxi-out time as the unimpeded taxi-out time [11]. The $15^{\text {th }}$ percentile is selected from the available literature. The $15^{\text {th }}$ percentile taxi out time is represented in red in each graph and it is 9, 7, 3 minutes unimpeded taxi-out time for respectively heavy, medium and light aircraft at BIA.

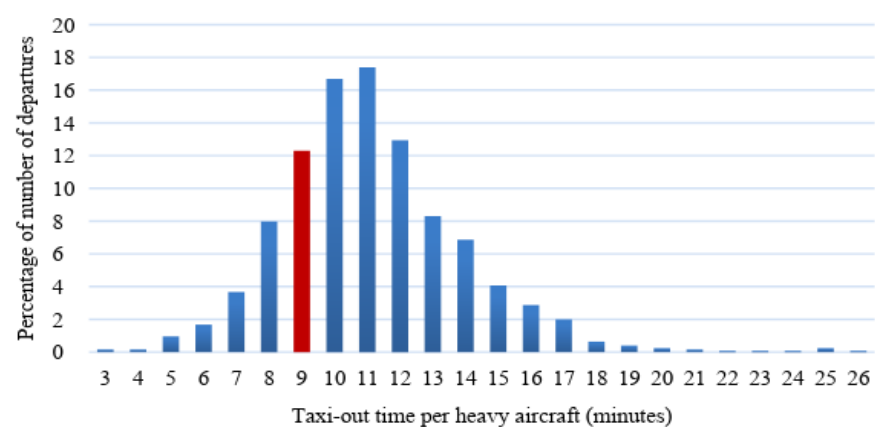

Fig. 3. Taxi-out time of Heavy aircraft

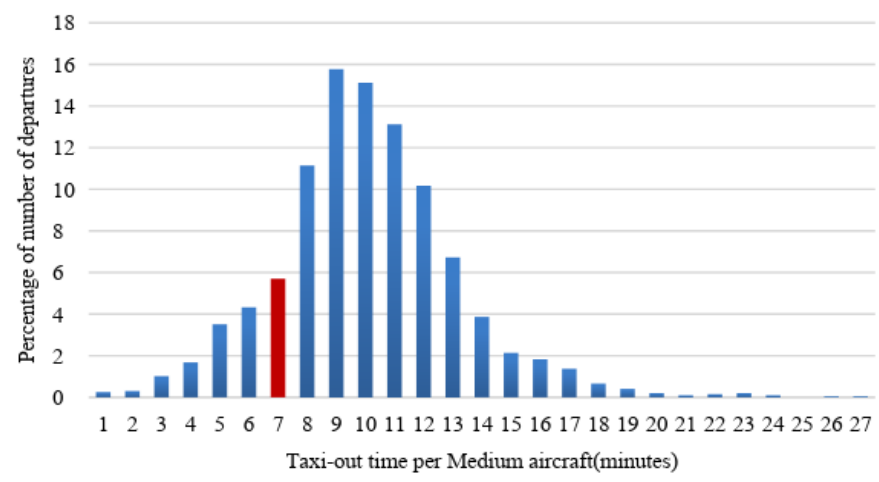

Fig. 4. Taxi-out time of medium aircraft

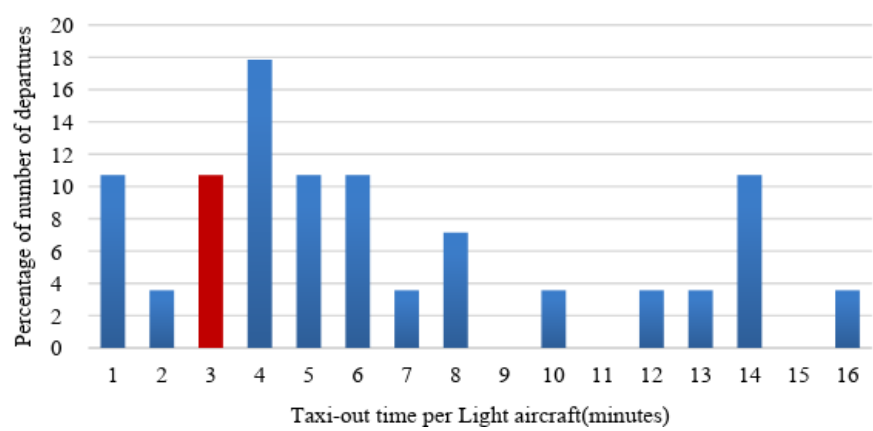

Fig. 5. Taxi-out time of light aircraft

\section{Estimating flight mix at BIA}

Monthly aircraft mix is calculated and assumed that percentages of monthly flight mix are consistent throughout the year. There are 38\% heavy flights, 57\% medium flights and $5 \%$ light flights are using BIA in the month of data analyzed.

It is assumed that delay percentages according to the aircraft type for the month of data analyzed, is consistent throughout the year. With the use of that assumption monthly delayed flights were calculated according to flight type.

Table I explains delay per flight, extra fuel consumption per flight and emission due to delay per flight according to aircraft type. When calculating per flight delay, delay time was given weight according to the number of aircraft percentage.

TABLE I

SUMMARY FOR THE MONTH OF DATA ANALYZED

\begin{tabular}{lccc}
\hline \hline & Heavy & Medium & Light \\
\hline Departure Delay (minutes/flight) & 3.30 & 4.00 & 5.04 \\
Fuel of Departures(liter/flight) & 110.71 & 48.27 & 11.89 \\
Emission of Departures(kg/flight) & 348.76 & 151.82 & 38.31 \\
\hline \hline
\end{tabular}

Then monthly departure flight delay, monthly excess fuel consumption of departure flights due to ground delay and emission aroused due to ground delay are calculated using Table I as the monthly number of flights are available[10]. Figure 6 depicts the estimated excess fuel usage in liters due to departure flight delays at ground operations. Figure 7 depicts the estimated emission aroused due to departure flight delays at ground operations.

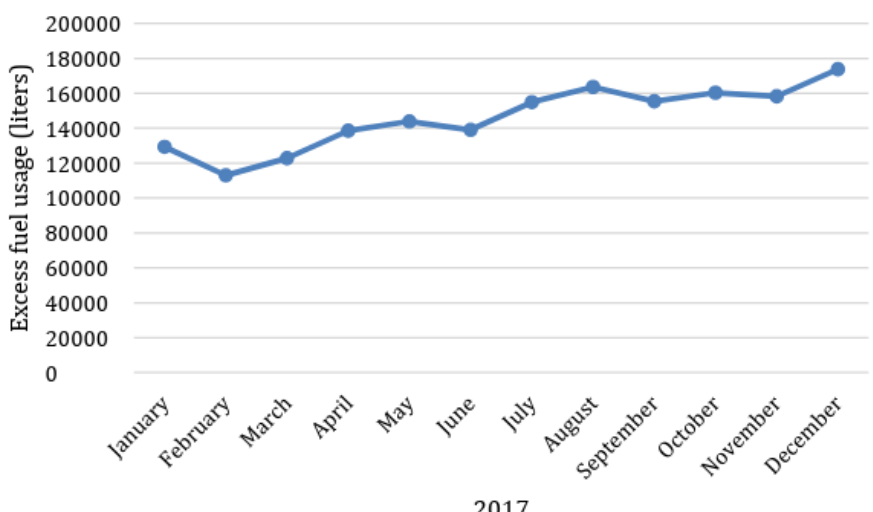

Fig. 6. Excess fuel usage due to the delay of departure flights at ground operations

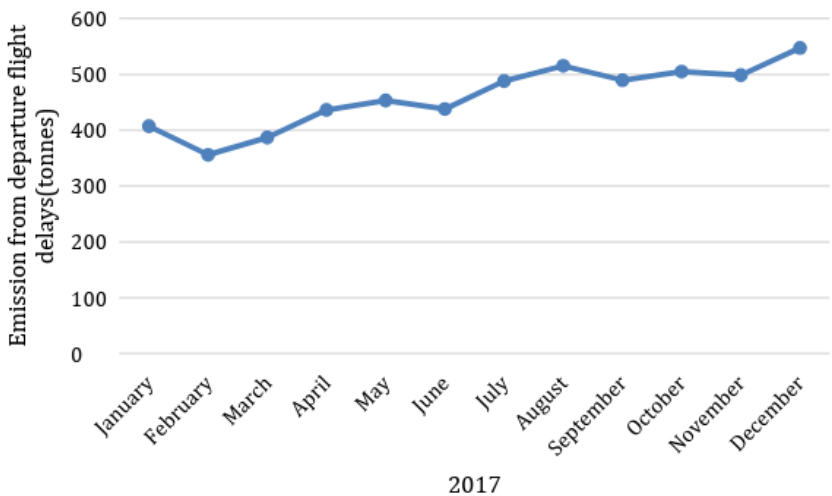

Fig. 7. Emission aroused due to delay of departure flights at ground operations 


\section{CONCLUSION}

Departure flight delays at ground movements operations result in considerable airport emission. Airport congestion, adverse weather, mechanical problems, inefficiencies in operations etc. cause for taxi-out delay. In fact, the emission of the taxiing phase is less than compared to all the other flight phases and it cannot be negligible when reaching towards green aviation.

Even though the aviation demand indicates a rapid growth, development of airport infrastructure has lagged behind it. Therefore, the demand of the airport exceeds planned capacity causing airport delays. BIA also faces the consequences of restricted capacity.

In 2017, the emission due to departure flight delays is estimated at 5520 tones while excess fuel usage is estimated at $1,752,937$ liters. When the demand is increasing BIA could face more delays and more emission in the future.

The study was able to quantify the departure flight delays at ground operations and its carbon emission for a small to medium scale airport with a single runway. As the airports get busier due to high demand, the delay tends to increase exponentially. Thus, the environmental impact of delay is several times greater than the case studied in this paper. Furthermore, this study only quantified the $\mathrm{CO}_{2}$ component of GHG. Even though there is a minor impact of other GHG components in comparison to $\mathrm{CO}_{2}$, those gases will also certainly increase the current estimate of delay related environmental impact.

The study outcomes can be used to make the relevant parties aware of the emission that they are responsible for. Excess fuel usage due to delay can be used as a measure for airlines' fuel efficiency and methods can be introduced to improve fuel efficiency in order to reduce aviation fuel cost. Emission reduction methods can be initiated and this study can be used as a reference at the implementation of emission reduction methods. This research was conducted for a small, single runway, capacity constrained airport and this method can be applied to any other airport in order to calculate their emission levels.

This research can be improved to find out baseline emission level at the airport. Once the baseline emission is calculated the emission reduction targets can be introduced. Following activities to mitigate emission improves the international image of the airport other than direct economic and environmental benefits. Thus, this research can be used as a guide to achieve green airport.

\section{ACKNOWLEDGMENT}

The authors would like to thank Mr. Sumith Tennakoon, Mr. Thilina De Silva, Air traffic controllers at Airport\& Aviation Services (Sri Lanka) Limited for providing guidance to gather data. We also grateful to acknowledge the Senate research committee for financial support. The research study was funded by the Senate Research Council Grant by the University of Moratuwa

\section{REFERENCES}

[1] K. D. Kuhn, "Ground delay program planning: Delay, equity, and computational complexity", Transp. Res. PART C, vol. 35, pp. 193203, 2013.

[2] "ACI has released the World Airport Traffic Forecasts 2016-2040.," 2016. [Online]. Available: http://www.aci.aero/News/Releases/MostRecent/2016/09/26/ACI-has-released-the-World-Airport-TrafficForecasts-20162040-marking-the-beginning-of-the-ACINAWorldAnnual-General-Assembly-Conference-and-Exhibition. [Accessed: 23Mar-2018].

[3] IPCC, "Climate Change 2014 Synthesis Report", Geneva, 2015.

[4] "Reducing emissions from aviation | Climate Action," 2017. [Online]. Available: https://ec.europa.eu/clima/policies/transport/aviation_en. [Accessed: 22-Mar-2018].

[5] C. N. Glover and M. O. Ball, "Stochastic optimization models for ground delay program planning with equity-efficiency tradeoffs", Transp. Res. Part C Emerg. Technol, 2013.

[6] EUROCONTROL, "Performance Review Commission", U.S./Europe Comparison of 2010 ATM-Related Operational Performance, 2010.

[7] EUROCONTROL, Airport Capacity Assessment Methodology, ACAM manual (4), 2016.

[8] ACI, "Airport Carbon and Emissions Reporting Tool (ACERT)", [Online]. Available: http://www.aci.aero/About-

ACI/Priorities/Environment/ACERT. [Accessed: 23-Jun-2018].

[9] AASL, "Airport \& Aviation Services (Sri Lanka) Ltd Annual Report", 2014.

[10] Ministry of Transport \& Civil Aviation Sri Lanka, "Movements in Bandaranaike International Airport (BIA)," 2018. [Online]. Available: http://www.transport.gov.lk/web/index.php/en/statistics/civil-aviationauthority.html. [Accessed: 19-Jul-2018].

[11] CANSO, Methodologies for Calculating Delays/Improvement Opportunity Pools By Phase of Flight, 2013.

\section{LIST OF ACRONYMS}
ACI - Airport Council International
CTOT - Calculated time of take-off
DAMR - Daily Aircraft Movement Record
GHG - Greenhouse gases
GMT - Greenwich Mean Time
ICAO - International Civil Aviation Organization
BIA - Bandaranaike International Airport. 